\title{
Oralismo versus lengua de signos: políticas de inclusión educativa sobre alumnado con deficiencia auditiva en Italia y España
}

Lopez-Torrijo, Manuel

Universidad de Valencia, Valencia, España

lopezm@uv.es

Garcia-Garcia, Fran J.

Universidad de Valencia, Valencia, España

garfran6@uv.es

\author{
Santana Hernández, Rafael \\ Universidad de Las Palmas de Gran Canaria, Las Palmas de Gran Canaria, España \\ rafael.santana@ulpgc.es
}

\section{Resumen}

A partir del Informe Warnock de 1978 la mayoría de los países europeos iniciaron procesos de integración, y posteriormente inclusión educativa, con un cierto desarrollo normativo. Gran parte de dicha legislación tiene un carácter general. De entre las múltiples necesidades y diversidades funcionales, tan sólo la supresión de barreras arquitectónicas ha tenido un tratamiento diferenciado. Recientemente han aparecido normativas específicas para la atención educativa y social a personas con deficiencia auditiva. Con motivo de analizar las últimas modificaciones se ha comparado la normativa referida a este colectivo en dos de los países con mayor índice de inclusión educativa de Europa: Italia y España. Las variables de comparación seleccionadas han sido: los objetivos; las personas afectadas; los derechos demandados; las necesidades del colectivo; los principios básicos de actuación; el sistema de comunicación; y los recursos que se ofrecen. La mayoría de familias con hijos con pérdida auditiva demandan una comunicación oral, siempre que sea posible. Los resultados de la investigación demuestran, además, los beneficios sobresalientes de la legua oral y de las ayudas técnicas. Sin embargo, la última legislación regula una oferta paralela de oralismo y lengua de signos en el caso de España y un enfoque casi exclusivo de lengua de señas en el caso de Italia, en contra de la investigación y las demandas de las familias. Se discuten las implicaciones educativas de ambas regulaciones, el contraste con el principio de equidad y su proyección social para la plena inclusión.

\section{Abstract}

In the wake of the Warnock Report in 1978 most of the European countries started up integration processes, and later inclusive education reforms, with a certain normative development. Much of this legislation has a general approach. Among several needs and functional diversities, only removal of architectural barriers has been proposed in a differentiated manner. Recently, specific regulations have appeared for the educational and social care of people with hearing impairment. In order to analyze the latest modifications, the regulations regarding this people have been compared in two of the countries with the highest educational inclusion rates in Europe: Italy and Spain. The comparison variables selected were: the objectives; the people subject to the regulations; the claimed rights; the needs of this collective; the basic principles of action; the communication mode; and the resources. Generally, families with children with hearing loss demand the oral communication, whenever possible. Research prove, in addition, the outstanding benefits of oral language and technical support. However, the latest legislation regulates a parallel offer of oral language and sign language in the case of Spain and an almost exclusive sign language approach in the case of Italy, against research and the demands of families. The educational implications of both regulations, the contrast with the principle of equity and the social projection for full inclusion are discussed.

Palabras clave: educación inclusiva, deficiencia auditiva, legislación educativa, lengua de signos, lengua oral, Italia, España.

Keywords: inclusive education, hearing impairment, educational legislation, sign language, oral language, Italy, Spain. 


\section{INTRODUCCIÓN}

La educación inclusiva (EI) constituye uno de los ejes fundamentales de la práctica y teoría pedagógicas actuales, amén de un planteamiento existencial. Es bien conocido que tal propuesta supone una progresión a partir de la etapa de educación especial y tras la integración educativa (Berruezo, 2009; López Torrijo, 1999) y que, como tales, ha sido liderada por la atención educativa de los entonces denominados minusválidos - discapacitados - alumnos con diversidad funcional.

Han sido las reivindicaciones de las familias, junto al compromiso ejemplar de algunos profesionales, quienes han hecho posible este proceso hacia la igualdad de oportunidades, la no-discriminación, la equidad y el disfrute de la diversidad. De hecho, estos logros han jalonado el reconocimiento de sus derechos en diversos documentos inter y supranacionales (Tabla 1).

Cada colectivo de personas con discapacidad ha reivindicado el derecho a una integración social plena mediante una atención educativa personalizada y acorde con sus necesidades.

El presente trabajo intenta profundizar en los últimos logros alcanzados por las personas con sordera, discapacidad auditiva y sordoceguera a nivel legislativo mediante un estudio comparado con dos países, cuyas peculiaridades lingüísticas ofrecen más posibilidades de normalización a estos colectivos y cuyos logros en El son una referencia europea e internacional: Italia y España.

Tabla 1. Documentos internacionales y europeos para la El.

\begin{tabular}{|c|c|c|}
\hline Fecha & Organismo & Documento \\
\hline Diciembre de 1971 & Naciones Unidas & Declaración de los Derechos de los Impedidos \\
\hline Marzo de 1990 & Unesco & Conferencia Mundial sobre Educación para Todos \\
\hline Junio de 1991 & Unesco & Declaración de Salamanca \\
\hline Diciembre de 1997 & Parlamento Europeo & Carta de Luxemburgo \\
\hline Marzo de 2000 & Unesco & Foro Consultivo Internacional sobre Educación para Todos \\
\hline Mayo de 2001 & OMS & Clasificación Internac. de Funcionamiento, Discapacidad y Salud \\
\hline Marzo de 2002 & Congreso Europeo & Declaración de Madrid \\
\hline Diciembre de 2006 & Naciones Unidas & Convención sobre Derechos de las Personas con Discapacidad \\
\hline Noviembre de 2008 & Unesco & Conferencia Internacional sobre Educación, $48^{\circ}$ sesión \\
\hline Mayo de 2014 & Unesco & Acuerdo de Mascate \\
\hline Mayo de 2015 & Unesco & Declaración de Incheón \\
\hline Noviembre de 2017 & Comisión Europea & Pilar Europeo del Derecho Social \\
\hline
\end{tabular}

Fuente: elaboración propia.

\section{MÉTODO}

\section{Unidades de comparación}

Las unidades de comparación fueron: la Legge quadro sui diritti di cittadinanza dlle persone sorde, con disabilitá uditiva in genere e sordocieche del 3 de octubre de 2017 y la Ley 27/2007, de 23 de octubre, por la que se reconocen las lenguas de signos españolas y se regulan los medios de apoyo a la comunicación oral de las personas sordas, con discapacidad auditiva y sordociegas (BOE 255 de 24 de octubre de 2007). 


\section{Variables de comparación}

Las variables de comparación fueron las categorías establecidas en las legislaciones nacionales:

- Objetivos

- Personas afectadas

- Derechos y necesidades reconocidos

- Ámbitos de aplicación

- Principios básicos de aplicación

- Sistemas de comunicación

- Recursos

\section{RESULTADOS}

\section{Objetivos}

Mientras que la normativa española se centra prioritariamente en «reconocer y regular la lengua de signos española», catalana y sucesivas autonómicas, y la «regulación de los medios de apoyo a la comunicación oral» (art. 1); el enfoque italiano parte de un reconocimiento fundamental de los derechos de las personas sordas, con discapacidad auditiva y sordociegas a su pleno desarrollo y participación social (art. 1.1). Italia asegura todos los recursos dedicados a la prevención y al cuidado de la sordera y sordoceguera, así como a la inclusión social y al acceso a la información, con una referencia expresa a la «sottotitulazione, servici di interpretariato in LIS e LIS tattile e ogni altra azione» (art. 1.2).

Tiene un valor especial el enfoque dedicado en Italia a las lenguas vehiculares. Apuestan por un bilingüismo completo: «Fermo restando l'insegnamento della lingua italiana parlata e scritta, riconosce, promuove e tutela la lingua dei segni italiana (LIS), in un'ottica di bilinguismo tra la lingua italiana parlata e scritta e la LIS, e la LIS tattile.»(art. 1.2).

\section{Personas afectadas}

Ambas normativas se refieren a las personas sordas, con discapacidad auditiva en general y sordociegas. (art. 4, a en España y art. 1 en Italia).

España diferencia como «signantes» a quienes utilizan la lengua de signos -LSE- (art. 4,a), frente a las «personas sordas, con discapacidad auditiva y sordociegas oralistas» (art. 4,b); afectados todos con una discapacidad $\geq 33 \%$ (art. $4, d$ ).

\section{Derechos y necesidades reconocidos}

El derecho fundamental que subrayan ambas normativas es la «libera scelta» o libre opción. España lo refiere «al aprendizaje, conocimiento y uso de las lenguas de signos españolas, y a los distintos medios de apoyo a la comunicación oral» (art. 2); y ello para ejercer efectivamente los derechos y libertades constitucionales: «el libre desarrollo de la personalidad, la formación en el respeto de los derechos y libertades fundamentales, el derecho a la educación y la plena participación en la vida política, económica, social y cultural» (art. 3.2). En la introducción se reconoce que no siempre estas personas pueden acceder a la información y a la comunicación por no disponer de intérpretes, de adaptaciones visuales y acústicas, o de los apoyos necesarios. 
En Italia especifican la aplicación de dicha opción al referirla expresamente: «alle modalità di co-municazione, ai percorsi educativi e agli au-sili utilizzati per il raggiungimento del pieno sviluppo della persona e della sua piena in-clusione sociale, provvedendo alle garanzie necessarie affinché le persone sorde, con di-sabilità uditiva in genere e sordocieche pos-sano liberamente fare uso della LIS, della LIS tattile e dei mezzi di sostegno alla co municazione in tutti gli ambiti pubblici e privati» (art. 2.1).

\section{Principios básicos de acción}

La legislación española dedica un apartado específico en el art. 5. a lo que denomina «principios generales». Con ellos fija la filosofía de actuación de las medidas concretas que va a desarrollar, indicando su sentido y proyección:

- 1. Transversalidad. Las políticas no deben limitarse a planes y acciones específicos, sino también a las políticas y líneas de acción de carácter general en cualquiera de los ámbitos de actuación pública, atendiendo las diversas necesidades y demandas de los usuarios.

- 2. Accesibilidad Universal. Los entornos, procesos, bienes-productos y servicios, objetos e instrumentos, herramientas y dispositivos deben cumplir las condiciones necesarias para ser comprensibles y utilizables por los usuarios con autonomía, seguridad y comodidad.

- 3. Libertad de elección. La «no discriminación» se recoge con términos casi idénticos en los dos países; en España se destaca que ninguna persona puede ser discriminada por su libre elección (art. 2.2 de Italia y art. 5, d en España).

- 4. Normalidad. Es el derecho a poder llevar una vida normal, accediendo a los lugares, ámbitos, bienes y servicios que accede cualquier persona.

\section{Sistemas de comunicación}

Los sistemas de comunicación planteados en las legislaciones de ambos países coinciden en diferencias las dos modalidades: la lengua de signos y la comunicación oral, mediante los correspondientes medios de apoyo. Italia no especifica el término «oral», dejando abierta e imprecisa dicha comunicación (art. 2.1). Sin embargo sí se amplía la opción «signante» diferenciando: «LIS, della LIS tattile» para personas con sordoceguera (art. 2).

España dedica la mayoría del texto a diferenciar los dos sistemas de comunicación mediante dos Títulos paralelos. El primero se refiere a la lengua de signos. El segundo no se refiere directamente a la comunicación oral, sino a lo que denomina «Aprendizaje, conocimiento y uso de los medios de apoyo a la comunicación oral». Cada uno de ellos se divide en dos capítulos: aprendizaje/conocimiento y uso.

\section{Recursos}

La legislación italiana no diferencia entre quienes usan lengua de signos y oral; los integra en un servicio global. Esta distinción imposibilita un estudio con paralelismos inmediatos entre países, pero sí se hizo entre las dos modalidades ofrecidas en España (Tabla 2 y 3 ).

Finalmente se determinan otras medidas de carácter general: creación en el plazo de un año de una Comisión de Seguimiento, con participación de las organizaciones de personas sordas y sus familias; compromiso de promover los «recursos humanos, técnicos y económicos necesarios para cubrir las medidas de acción positiva» (Dis. Adic. Segunda); estudio de la población con sordoceguera para establecer los centros de recursos precisos para su atención; aportar financiación para la adquisición de apoyos técnicos; creación de sendos reglamentos para la utilización de la LSE y la eliminación de barreras de comunicación y la integración social de estos colectivos. 
Tabla 2. Recursos para aprendizaje/conocimiento en España.

\begin{tabular}{cc}
\hline \multicolumn{1}{c}{ Lengua de signos (arts. 7-8) } & Comunicación oral (arts. 16-17) \\
\hline LSE & Lormación reglada \\
Modelos educativos bilingües \\
LSE (asignatura optativa) \\
Titulaciones de intérpretes \\
Formación para profesorado. & Titulaciones de profesores de apoyo \\
Formación no-reglada & Formación para profesorado. \\
\hline $\begin{array}{c}\text { Cooperación entre familias, instituciones educativas, asociaciones } \\
\text { de personas sordas: cursos LSE. }\end{array}$ & ídem. \\
$\begin{array}{c}\text { Cooperación entre Administraciones públicas, Universidades, } \\
\text { asociaciones de personas sordas: aprendizaje de la LSE. }\end{array}$ & ídem. \\
\hline
\end{tabular}

Fuente: elaboración propia.

La financiación de estos recursos es competencia del Ministerio de Trabajo y Asuntos Sociales, en colaboración con los Ministerios competentes. Hay una alusión explícita a la financiación por parte de la Administración General del Estado de «el fomento, la investigación y la difusión del uso de la lengua de signos catalana» (Disp. Final tercera).

A diferencia de la normativa española, la italiana declara desde los inicios y de forma global la relación de todos los servicios que debe prestar la República, siguiendo el proceso cronológico de las necesidades de este colectivo:

- Prevención y detección precoz: prenatal y con screening neonatal universal. (art. 3.1).

- Diagnóstico audiológico (art. 3.1).

— Intervención diagnóstica temprana, hablitadora y rehabilitadora (art. 3.1).

- Prótesis auditivas y oculares precoces, los implantes cocleares y la logopedia (art. 3.1).

- Atención y apoyo psicológico, informativo y pedagógico para niños y familias (art. 3.2).

- Accesibilidad tecnológica (art. 3.4).

- Accesibilidad a la comunicación, información, espacios públicos (art. 4.1 y 4.4).

- Sistemas alternativos a la comunicación hablada, la LIS o la subtitulación (art. 4.3).

- Servicios de emergencia, alarmas y acontecimientos excepcionales (art. 4.5).

- Acceso a la Administración mediante «metodología comunicativa e inclusiva» (art 4.6).

- Inclusión educativa, mediante los servicios «dell'insegnante di sostegno, dell'assistente alla comunicazione nel caso di alunni sordi e dell'assistente all'autono-mia e alla comunicazione nel caso di alunni sordociechi, dell'interprete in LIS e LIS tat-tile». Igualmente, mediante las ayudas tecnológicas y otros medios que garantizan la plena participación y accesibilidad a las actividades escolares y extraescolares (art. 5.1). Para asegurar los stándares de calidad de los intérpretes y asistentes a la autonomía y la comunicación, así como para la convalidación de los títulos anteriores, se creará en el plazo de 90 días una Comisión de expertos y representantes de las asociaciones, coordinada por el Ministerio de Educación (art. 5.3).

- Formación universitaria y post-universitaria: suprimiendo las barreras de comprensión y comunicación con medidas como estenotipia, respeakeraggio, LIS, LIS tattile, recursos informáticos, etc., que aseguren la igualdad de oportunidades y la autonomía (art. 6).

— Inclusión laboral y formación permanente: consiguiendo la igualdad de oportunidades y la accesibilidad total mediante el uso de LIS, LIS táctil, TICs y aplicaciones de chat, de e-mail, videoconferencias, etc. (art 7).

- Protección de la salud: a través de la comunicación e información que puedan aportar los intérpretes, el uso de cualquier canal comunicativo y lingüístico, tecnologías, etc., especialmente en lo referente a las campañas informativas y de prevención (art. 8).

- Arte, cultura y tiempo libre: acceso al patrimonio, turismo, deporte y recreación mediante los servicios anteriormente reiterados (art. 9).

- Participación política mediante los recursos citados anteriormente y, en particular, en las sesiones plenarias de todo estamento político se promoverá la interpretación a LIS, LIS táctil y el subtitulado (art. 10). 
El plazo de aplicación de la presente ley italiana y de sus consiguientes reglamentos relativos al Ministerio de Trabajo y Asuntos Sociales se limitará a 3 meses a partir de la publicación de la ley, y contará con el asesoramiento de los Ministerios afines, la Universidad, las asociaciones de personas afectadas y las asociaciones de profesionales relacionados (art. 11.1). El plazo de los temas educativos será de 6 meses (art. 11.2).

Se creará un grupo específico para tratar los aspectos relacionados con la sordera, discapacidad auditiva y sordoceguera dentro del Observatorio Nacional sobre la Condición de las Personas con Discapacidad. Dicho grupo promoverá: un plan de actuación bienal; un estudio estadístico territorial del colectivo; y valorará la aplicación de la normativa. Por su parte, el gobierno enviará un informe bienal a las Cámaras sobre el desarrollo de esta ley.

Sin embargo, todos los planteamientos teóricos enumerados pierden efectividad con la limitación económica que cierra la normativa al señalar que se proveerán los medios «nei limiti delle risorse umane, strumentali e finanziarie disponibile a legislaciones vigente e comunque sensa nuovi o maggiori oneri a carico della finanza pubblica» (art. 14). Como es evidente, los fondos reservados en el ejercicio anterior para otros fines son, de hecho, para otros fines y no para los que recoge la nueva legislación.

Tabla 3. Recursos para el uso de medios de comunicación en España.

\begin{tabular}{cc}
\hline Lengua de Signos (arts. 9. 15) & Comunicación oral (arts. 18-24) \\
\hline Servicios de intérpretes de LSE & Uso de los medios de apoyo a la comunicación oral \\
Medidas contra la discriminación y de acción positiva. & ídem. (Inclusive atención a personas sordociegas) \\
\hline
\end{tabular}

Educación

LSE, lengua vehicular

Intérpretes de LSE

Asesoramiento y apoyo en la Universidad

Lengua oral

Medios de apoyo a la comunicación oral ídem.

Formación y empleo

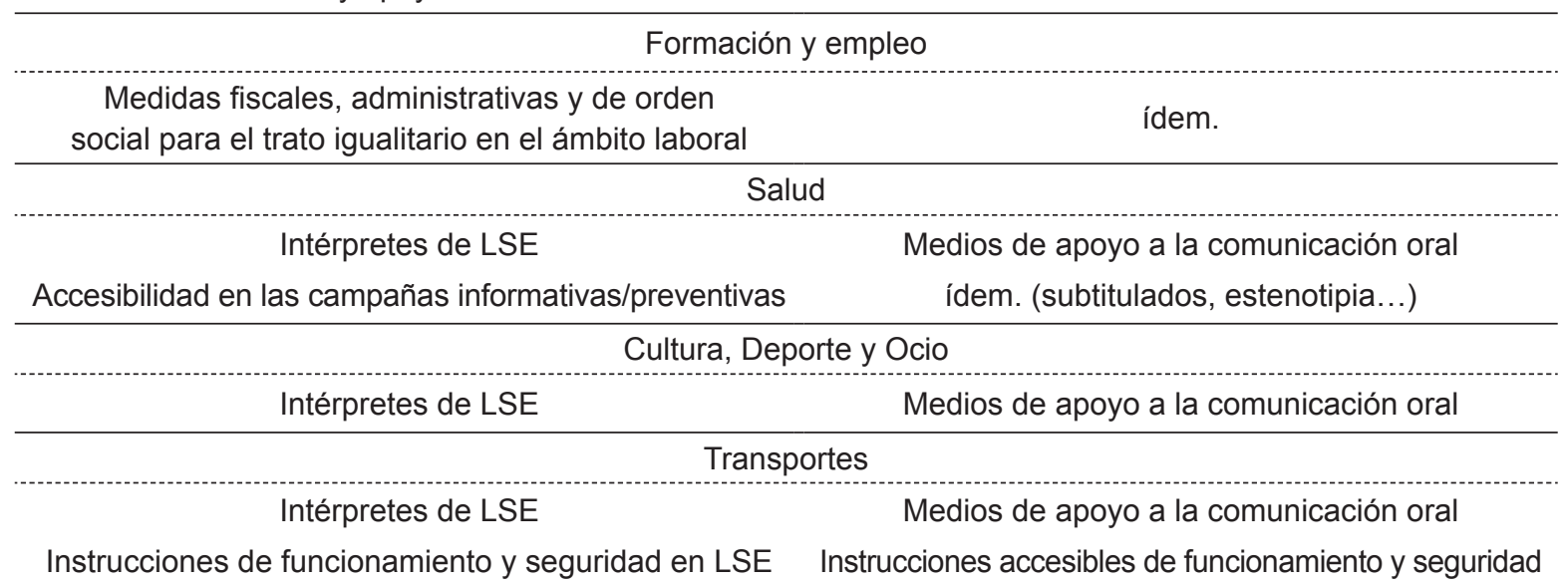

\begin{tabular}{|c|c|}
\hline \multicolumn{2}{|c|}{ Relaciones con las Administraciones públicas } \\
\hline Intérpretes de LSE & Formación y disponibilidad \\
\hline \multicolumn{2}{|c|}{ Participación política } \\
\hline Intérpretes de LSE & Accesibilidad con medios de apoyo a la com. oral. \\
\hline \multicolumn{2}{|c|}{ Medios de comunicación social, telecomunicaciones y sociedad de la información } \\
\hline \multicolumn{2}{|l|}{ Intérpretes de LSE } \\
\hline Accesibilidad Universal (alarmas, excepción y sitio) & ídem. \\
\hline Incentivos para acceso a telecomunicaciones en LSE & Incentivos para acceso oral a telecomunicaciones \\
\hline Centro de Normalización Lingüística de la LSE & Centro Español de Subtitulado y la Autodescripción \\
\hline Investigar, fomentar y velar por el buen uso de la LSE. & $\begin{array}{l}\text { Investigar, fomentar, coordinar actuaciones y } \\
\text { extender la subtitulación y autodescripción. }\end{array}$ \\
\hline Consultas y convenios con Universidades & (No se alude a los profesionales de dicho Centro). \\
\hline y asociaciones de personas sordas. & \\
\hline $\begin{array}{l}\text { Gestión paritaria entre la Administración y el } \\
\text { movimiento asociativo de personas usuarias de la LSE. }\end{array}$ & - \\
\hline
\end{tabular}

Fuente: elaboración propia. 


\section{DISCUSIÓN Y CONCLUSIONES}

El análisis de las normativas presentadas señala una evidente diferencia en cuanto al enfoque de las mismas. La legislación española refleja desde la primera línea una prioridad manifiesta por el reconocimiento y regulación de la lengua de signos española y catalana. Ello es fruto de la presión asociativa que se realizó desde la Confederación Estatal de Personas Sordas (CNSE). El legislador asume en su introducción aquella manipulación histórica y todas las imprecisiones psicolingüisticas que le dictó la CNSE (López Torrijo, 2005).

A ello se opusieron las demandas mayoritarias de las familias con hijos con sordera a través de su máximo órgano nacional de representación: la Confederación Española de Familias de Personas Sodas (FIAPAS). Apostaron mayoritariamente por una comunicación oral. El resultado es una normativa que, con una intención "salomónica», resulta casi esperpéntica al ofrecer una presentación formal en las que el legislador va repartiendo a uno y otro bando formulaciones casi idénticas y en las que casi sólo cambia el epíteto final de: «mediante la lengua de signos y los intérpretes de lengua de signos» o «mediante los medios de apoyo a una comunicación oral».

Frente a este enfoque, Italia presenta una ley que integra de forma global todos los servicios precisos de forma abierta a las aportaciones futuras y siguiendo las exigencias que se producen a lo largo del proceso vital de estas personas. Son especialmente destacables su incorporación del diagnóstico temprano, las aportaciones tecnológicas de los implantes cocleares, los apoyos psicopedagógicos a los niños y familiares afectados, el acceso a la lengua italiana hablada y escrita, amén de todos los recursos necesarios para una Accesibilidad Universal a todos los servicios educativos, sociales, jurídicos, de transporte, comunicación, así como a una autonomía personal y participación social y política plenas.

Los derechos residen en las personas, más allá de los consensos que acordemos en nuestra convivencia, Pero es vital que tales derechos se vean plasmados y reconocidos en la legislación que la regula. Desde esta perspectiva, ambas normativas suponen un avance importante en el reconocimiento de los derechos de las personas con sordera. Igualmente crucial es la concreción de los servicios que se les asegura aportar para su desarrollo en igualdad y que hemos enumerado esquemáticamente. Sin embargo, tales enunciados son meros eufemismos, porque no son exigibles. En el caso de la normativa española, los verbos que concretan el cumplimiento y exigibilidad de tales servicios son los siguientes: «propiciará» (art. 7,4 y16,3), se «facilitará» (arts. 7,1; 10 , a; 12,$1 ; 13,1 ; 14,5 ; 16,1 ; 19$, a); se «fomentará» (arts. 8,1 y 17,1); «promover la prestación de servicios» (arts. 9; 10, a, c y d; 12, 1 y $2 ; 13,2 ; 14,5 ; 15,3 ; 18 ; 19$,a,c y d; $21 ; 22,2 ; 23,1$ y 5 ); siempre que sea posible (art 11, 2); incentivar (art 14, 3); prestar servicios «donde se determine» (arts. 7,$2 ; 11$ ) y 20 ; cooperar (arts. 8,1 y $2 ; 17,2$ ). En el caso de Italia, se aplica la limitación económica para poder asegurar tales servicios, pero sin aumentar los presupuestos actuales.

Sería preciso que el legislador tuviera presente, antes de delimitar los servicios, las aportaciones que en fechas recientes ofrece a este colectivo la medicina (prevención y detección temprana, soluciones auditivas implantables mediante cirugía en cóclea, zona ósea o tronco cerebral, etc.), la tecnología (audífonos digitales de última generación, implantes cocleares, bucles magnéticos, emisoras de frecuencia modulada, bluetooth, etc.), la psicolingüística, la logopedia, la pedagogía, y un largo etcétera de formas que aseguran la autonomía de las personas y una participación social plena (Moreno-Torres, Cid, Santana, y Ramos, 2011; Santana y Torres, 2013). En cualquier caso, convendría ser conscientes de las consecuencias que se derivan de cada uno de los dos enfoques comunicativos y respetar siempre las graves limitaciones de algunas pérdidas auditivas, además de los contextos donde se desarrollan.

A este diálogo interdisciplinar convocamos especialmente a los especialistas en derecho, a fin de que ayuden a conjugar los derechos y obligaciones constitucionales a conocer y utilizar las lenguas oficiales -siempre que la discapacidad lo posibilite- con las exigencias de sistemas, recursos y servicios alternativos, no siempre conducentes al ejercicio de los derechos plenos de todo ciudadano. La traslación de esta propuesta a otros colectivos (inmigrantes, demandas políticas,...) podría clarificar las respuestas.

En cualquier caso, reiteramos que el derecho reside en las personas y sólo una mirada justa y solidaria puede alcanzar a desarrollarlo, más allá de las concreciones legales. 


\section{REFERENCIAS BIBLIOGRÁFICAS}

Berruezo, R. (Coord.) (2009). El largo camino hacia una educación inclusiva. Pamplona: UPNA.

Legge quadro sui diritti di cittadinanza dlle persone sorde, con disabilitá uditiva in genere e sordocieche del 3 de octubre de 2017.

Ley $27 / 2007$, de 23 de octubre, por la que se reconocen las lenguas de signos españolas y se regulan los medios de apoyo a la comunicación oral de las personas sordas, con discapacidad auditiva y sordociegas (BOE 255 de 24 de octubre de 2007).

López Torrijo, M. (1999). Textos para una historia de la Educación especial. Valencia: Tirant lo Blanc.

López Torrijo, M. (2005). La educación de las personas con sordera: La escuela oralista española. Valencia: Universitat de Valencia.

Moreno-Torres, I., Cid, M., Santana, R., y Ramos, A. (2011). Estimulación temprana y desarrollo linguiistico en niños sordos con implante coclear: el primer año de experiencia auditiva. Revista de Investigación en Logopedia, 1, 56-75.

Santana, R. y Torres, S. (2013). Necesidades y respuesta educativa a la discapacidad auditiva. Las Palmas de Gran Canaria: Universidad de Las Palmas de Gran Canaria. 